\title{
Modulating the Structure and Properties of Cell Membranes: The Molecular Mechanism of Action of Dimethyl Sulfoxide
}

\author{
Andrey A. Gurtovenko and Jamshed Anwar* \\ Computational Laboratory, Institute of Pharmaceutical Innovation, University of Bradford, \\ Bradford, West Yorkshire, BD7 1DP, U.K.
}

Received: April 23, 2007; In Final Form: June 11, 2007

\begin{abstract}
Dimethyl sulfoxide (DMSO) is a small amphiphilic molecule which is widely employed in cell biology as an effective penetration enhancer, cell fusogen, and cryoprotectant. Despite the vast number of experimental studies, the molecular basis of its action on lipid membranes is still obscure. A recent simulation study employing coarse-grained models has suggested that DMSO induces pores in the membrane (Notman, R.; Noro, M.; O’Malley, B.; Anwar, J. J. Am. Chem. Soc. 2006, 128, 13982-13983). We report here the molecular mechanism for DMSO's interaction with phospholipid membranes ascertained from atomic-scale molecular dynamics simulations. DMSO is observed to exhibit three distinct modes of action, each over a different concentration range. At low concentrations, DMSO induces membrane thinning and increases fluidity of the membrane's hydrophobic core. At higher concentrations, DMSO induces transient water pores into the membrane. At still higher concentrations, individual lipid molecules are desorbed from the membrane followed by disintegration of the bilayer structure. The study provides further evidence that a key aspect of DMSO's mechanism of action is pore formation, which explains the significant enhancement in permeability of membranes to hydrophilic molecules by DMSO as well as DMSO's cryoprotectant activity. The reduction in the rigidity and the general disruption of the membrane induced by DMSO are considered to be prerequisites for membrane fusion processes. The findings also indicate that the choice of DMSO concentration for a given application is critical, as the concentration defines the specific mode of the solvent's action. Knowledge of the distinct modes of action of DMSO and associated concentration dependency should enable optimization of current application protocols on a rational basis and also promote new applications for DMSO.
\end{abstract}

\section{Introduction}

Manipulating the structure and properties of cell membranes is an issue which is fundamental to numerous applications in the biosciences, pharmaceuticals, and biotechnology. Since the lipid bilayer serves as a structural framework for all other constituents of cell membranes, it is often considered as a primary target for modulation. For many applications, it is highly desirable that the membrane structure and properties are modulated in a controlled, and for specific applications in a reversible, manner.

Dimethyl sulfoxide (DMSO) is a small amphiphilic molecule with a hydrophilic sulfoxide group and two hydrophobic methyl groups (Figure 1). Its amphiphilic nature appears to be an important defining characteristic for its action on membranes: DMSO is widely employed in cell biology to induce cell fusion ${ }^{1}$ and cell differentiation. ${ }^{2}$ It is also an effective penetration enhancer ${ }^{3}$ and is routinely used as a cryoprotectant. ${ }^{4}$ While the protocols for each of these applications are well developed, our understanding of the molecular basis for DMSO's actions on cell membranes is still rudimentary, ${ }^{5}$ mainly due to limitations of current experimental techniques. Consequently, optimization of the protocols, e.g., identification of the optimal concentration of DMSO, is largely driven by empiricism. The lack of a molecular understanding for DMSO's action is probably also hindering the identification of new applications for this versatile solvent.

\footnotetext{
* Corresponding author.
}
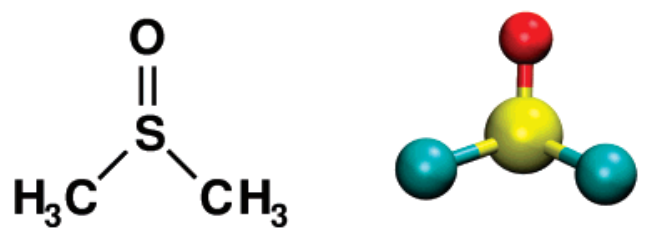

Figure 1. Chemical structure of a dimethyl sulfoxide (DMSO) molecule (left) and its united-atom representation used in this study (right).

A way forward is to resort to molecular simulation techniques that are now able to provide an unprecedented molecular insight into a wide range of phenomena; often molecular simulations are the only source of information at the atomic scale. Indeed, the interaction of DMSO with phospholipid membranes has been studied by molecular simulations. ${ }^{6-8}$ While a conclusive molecular-level explanation for its pharmacological activity is still lacking, the latest simulation study ${ }^{8}$ that employed coarsegrained models did suggest a credible explanation for some aspects of DMSO activity, namely, that DMSO induces pore formation in the membrane. In coarse-grained simulations a number of atoms are represented by a single particle, which makes the simulations computationally efficient, enabling longer time and length scales to be accessed but with a loss of chemical specificity. Clearly, it is essential to confirm whether the extraordinary feature of pore formation is observed in realistic atomic-level simulations where the chemistry is fully defined.

In this work we systematically studied the effects of DMSO on a phospholipid membrane in the liquid-crystalline phase using 

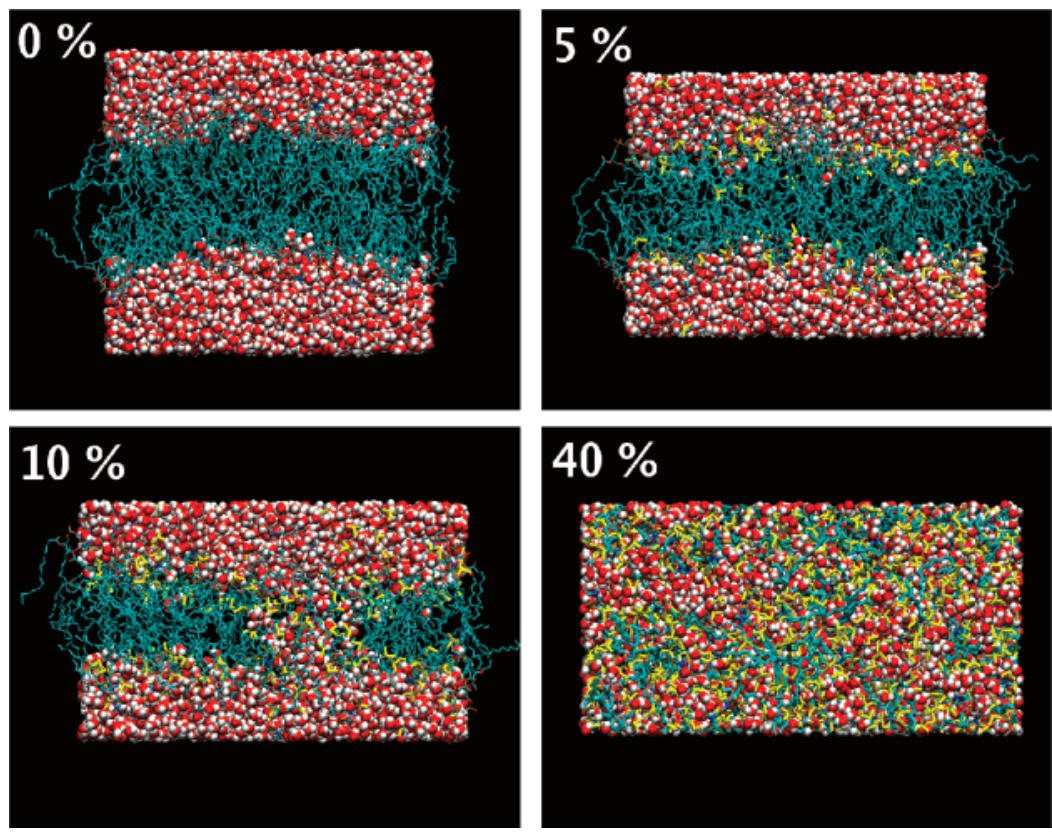

Figure 2. Distinct modes of action of DMSO on phospholipid membranes. Presented are side views of the final structures for the bilayer systems containing $0,5,10$, and 40 mol \% of DMSO (lipid-free basis). Lipids are shown in cyan, water in red, and DMSO in yellow.

atomic-scale molecular dynamics (MD) simulations with realistic potentials for the modeled molecules. We investigated 14 different concentrations of DMSO varying from 0 mol \% (pure water) to $100 \mathrm{~mol} \%$ (pure DMSO solvent). DMSO appears to exhibit three distinct modes of action on the phospholipid membrane, the specific mode being dependent on its concentration. These modes of action are summarized visually in Figure 2. Depending on concentration, DMSO is able to decrease the membrane thickness $(2.5-7.5 \mathrm{~mol} \%)$, induce formation of transient water pores $(10-20 \mathrm{~mol} \%)$, or destroy the bilayer structure of membranes $(25-100 \mathrm{~mol} \%)$. We discuss the results and their significance in detail below, beginning with validation of the molecular models.

\section{Methods}

Atomic-scale MD simulations were performed on lipid bilayers comprising zwitterionic dipalmitoylphosphatidylcholine (DPPC) lipids in aqueous solution with DMSO. The concentration of DMSO was systematically varied from $0 \mathrm{~mol} \%$ (pure water) to $100 \mathrm{~mol} \%$ (pure DMSO solvent). In all, we considered 14 different DMSO molar concentrations: $0 \%, 2.5 \%, 5.0 \%$, $7.5 \%, 10.0 \%, 12.5 \%, 15.0 \%, 20.0 \%, 25.0 \%, 30.0 \%, 35.0 \%$, $40.0 \%, 60.0 \%$, and $100.0 \%$ (lipid-free basis). In all simulations the DPPC bilayers comprised 128 lipid molecules; the total number of solvent molecules (water and DMSO) was fixed to 3655 so that the number of solvent molecules per lipid was constant $(\sim 29)$ for all systems. Periodic boundary conditions were applied in all three dimensions.

Force-field parameters for DPPC lipids were taken from the united-atom force-field of Berger et al. ${ }^{9}$ Water was modeled using the simple point charge (SPC) model. ${ }^{10}$ For DMSO the force-field developed by Bordat et al. ${ }^{11}$ was used. The LennardJones interactions were truncated at $1 \mathrm{~nm}$; the electrostatic interactions were handled using the particle-mesh Ewald (PME) method. ${ }^{12,13}$ The simulations were performed in the $N p T$ ensemble with semi-isotropic pressure coupling (isotropic in $\mathrm{x}$ and $\mathrm{y}$, and independent in the $\mathrm{z}$ direction). The temperature and pressure were controlled using the Berendsen thermostat and barostat, ${ }^{14}$ respectively. The pressure was set to 1 bar while the temperature was set to $350 \mathrm{~K}$, which is above the phase transition temperature of a DPPC bilayer in DMSO/water at all considered DMSO concentrations (we recall that DMSO has been observed to increase the phase transition temperature from a gel to a liquid-crystalline phase for DPPC-DMSO-water mixtures). ${ }^{15}$ Thus, the studied DPPC bilayers were in the fluid, liquid-disordered phase.

The time step used was 2 fs. All simulations were of $30 \mathrm{~ns}$ duration for each considered DPPC/DMSO/water system, except the system containing $10 \mathrm{~mol} \%$ of DMSO for which the simulation time was extended to $50 \mathrm{~ns}$ due to a slow pore formation process. Only the last $10 \mathrm{~ns}$ of each MD trajectory were used for a subsequent analysis. All simulations were performed using the GROMACS suite. ${ }^{16}$ The combined simulated time of all MD runs amounted to a half of a microsecond.

To elucidate possible effects of the periodic boundary conditions on our main findings, three additional simulations of DPPC bilayer systems with $2.5,10.0$, and $30.0 \mathrm{~mol} \mathrm{\%}$ of DMSO were performed, in which the number of solvent molecules (DMSO and water) were increased by a factor of 4 . Each of these simulations was run for $20 \mathrm{~ns}$ duration.

\section{Results}

A fundamental structural characteristic of lipid bilayers is the average area per lipid, $\langle A\rangle$, which can be measured accurately by experiment. ${ }^{17}$ For a DMSO-free DPPC bilayer the average area per lipid from the simulations was found to be $\langle A\rangle=0.690$ $\pm 0.002 \mathrm{~nm}^{2}$. This closely matches the experimental value of $0.719 \mathrm{~nm}^{2}$ reported for a DPPC bilayer at $T=353 \mathrm{~K},{ }^{18}$ thereby validating the model used. Adding a small amount of DMSO into a bilayer system (up to $10 \mathrm{~mol} \%$ ) leads to a pronounced increase in the area per lipid and a decrease in the closely related thickness of the bilayer, $d_{\text {membrane }}$ (see Figure 2 and Table 1 ). The mass density profiles for the various components of the system are shown in Figure 3. They reveal that DMSO molecules readily penetrate into the lipid/water interface of the membrane and occupy positions just beneath the lipid headgroups. This suggests that DMSO does not interact favorably with the lipid headgroups but has a greater affinity, by virtue of its methyl groups, toward the hydrocarbon lipid chains. The absence of interactions between DMSO molecules and lipid 
TABLE 1: Concentration-Dependent Modes of Action of DMSO on Phospholipid Membranes

\begin{tabular}{ccccl}
\hline system & $\begin{array}{c}C_{\text {DMSO }} \\
{[\mathrm{mol} \mathrm{\%}]^{a}}\end{array}$ & $\begin{array}{c}d_{\text {membrane }} \\
{[\mathrm{nm}]^{b}}\end{array}$ & $\begin{array}{c}\text { area per lipid } \\
{\left[\mathrm{nm}^{2}\right]}\end{array}$ & $\begin{array}{c}\text { DMSO mode } \\
\text { of action }\end{array}$ \\
\hline 1 & 0.0 & $3.62 \pm 0.01$ & $0.690 \pm 0.002$ & \\
2 & 2.5 & $3.42 \pm 0.01$ & $0.766 \pm 0.002$ & membrane \\
3 & 5.0 & $3.23 \pm 0.01$ & $0.848 \pm 0.002$ & thinning \\
4 & 7.5 & $3.05 \pm 0.02$ & $0.928 \pm 0.003$ & \\
5 & 10.0 & $2.35 \pm 0.03$ & & membrane \\
6 & 12.5 & $2.13 \pm 0.05$ & & thinning \\
7 & 15.0 & $1.53 \pm 0.06$ & & and \\
8 & 20.0 & $0.52 \pm 0.08$ & & pore formation \\
9 & 25.0 & & & \\
10 & 30.0 & & & \\
11 & 35.0 & & & membrane \\
12 & 40.0 & & & disintegration \\
13 & 60.0 & & & \\
14 & 100.0 & & &
\end{tabular}

${ }^{a}$ Molar concentration of DMSO (lipid-free basis). ${ }^{b}$ Membrane thickness measured as the average distance between phosphate groups of two leaflets.

headgroups are also seen through inspecting radial distribution functions (RDF) of DMSO atoms with choline, phosphate, and carbonyl groups of DPPC lipids (data not shown). Most of these RDFs (except one for choline groups) do not reveal a pronounced first peak, suggesting a lack of interaction between DMSO and the headgroups in the interior of water/lipid interface. Such a peak is observed for the choline groups and most likely associated with DMSO being present in the water phase but not in the membrane.

It is instructive to examine the influence of DMSO on the coordination of DPPC headgroups with each other at the lipid/ water interface of a bilayer. Pure, i.e., DMSO-free phosphatidylcholine bilayers demonstrate strong dynamic charge pairing between phosphate and choline groups. ${ }^{19}$ Therefore, the average coordination of phosphorus atoms with nitrogen atoms of lipid headgroups can be a useful tool for probing the properties of the lipid/water interface. ${ }^{20}$ The average coordination number of $\mathrm{N} \cdots \mathrm{P}$ interactions is plotted in Figure $4 \mathrm{a}$ and shows a dramatic drop with increase in DMSO concentration. Thus, DMSO, on penetration into the lipid/water interface, acts as a spacer between the lipids, increasing the average lateral distance between them and destroying the N $\cdots P$ pairing. The DMSOinduced expansion of the DPPC membrane accompanied by a concurrent decrease in the membrane thickness, $d_{\text {membrane, }}$ is tabulated in Table 1. The induced lateral expansion of lipids also appears to enable the lipid headgroups to reorientate and causes a decrease in the ordering of the acyl chains, thus making the membrane more fluid. In a DMSO-free bilayer, the lipid headgroups are oriented almost parallel to the bilayer surface due the above-mentioned pairing between the headgroups, the average angle $\alpha$ between the $\mathrm{P}-\mathrm{N}$ vector of a DPPC lipid and the outward bilayer normal being $80.2^{\circ} \pm 0.2^{\circ}$. DMSO, even at low concentrations, enables the headgroups to adopt a more vertical orientation with respect to the lipid/water interface (Figure 4b).

As DMSO concentration is raised to $10 \mathrm{~mol} \%$ and higher we observe further lateral expansion of the bilayer, which is accompanied by the progressive loss of interactions between the headgroups of different lipid molecules and by the related decrease in bilayer thickness (see Figure 4a and Table 1). As a result, the lipid/water interface becomes rather prone to structural defects resulting from thermal fluctuations. Furthermore, increasing numbers of DMSO molecules near the lipid/water interface force their appearance into the membrane interior:
DMSO molecules accumulate now not only at the interface but also in the hydrophobic core (Figure 3), interacting favorably with acyl lipid chains and screening effectively their hydrophobicity. All these factors favor entrance of water molecules into the membrane interior, leading to formation of transient water defects. These defects can be visualized as transient "water columns" or "water fingers" spontaneously spanning the entire membrane and disappearing within a few picoseconds. Because of the stochastic nature of these defects, some rather large water columns comprising a significant number of water molecules can develop in the membrane with time (Figure 5). Such water defects remain stable for a rather long time due to hydrogen bonding between the water molecules in the columns. The presence of water in the hydrophobic core of a lipid membrane, being energetically unfavorable, eventually leads to the redistribution of lipid headgroups from the water-lipid interface to the membrane interior, surrounding and stabilizing the water defect. In such a way we observe the remarkable feature of DMSO inducing the formation of water pores in the phospholipid membrane (Figures 2, 3, and 5). The overall picture of pore formation is reminiscent to that reported in MD simulations of electroporation in lipid membranes. ${ }^{21,22}$

The characteristic time of pore formation was found to be strongly dependent on DMSO concentration. The slowest pore formation ( $\sim 20 \mathrm{~ns})$ was observed for a DPPC bilayer with 10 mol \% of DMSO, the fastest one ( $\sim 3 \mathrm{~ns})$ for the bilayer system with $20 \mathrm{~mol} \%$ of DMSO. To characterize the process of pore formation, in Figure $4 \mathrm{c}$ we present the time evolution of the numbers of DMSO molecules, water molecules, and lipid headgroups in $0.5 \mathrm{~nm}$ slab in the center of the bilayer for the system with $12.5 \mathrm{~mol} \%$ of DMSO. The DMSO molecules rapidly partition into the membrane interior, reaching equilibrium concentration within the membrane in about 3 ns. This promotes formation of spontaneous water defects and subsequent reorientation of lipid headgroups toward the membrane interior, giving rise to pore formation. The number of water molecules in the center of the membrane is subject to considerable fluctuations, whereas the numbers of DMSO and lipid headgroups are relatively stable (Figure 4c). This is in part because of the presence of small, spontaneously occurring water defects in the membrane in addition to the relatively large and stable water pores as seen in Figure 5. The reorientation of lipid headgroups toward the membrane interior can also be monitored through the change of the angle $\alpha$ between the $\mathrm{P}-\mathrm{N}$ vector of a lipid headgroup and the outward bilayer normal (Figure 4b). The average angle $\langle\alpha\rangle$ for the bilayer system with a pore (10 mol \% of DMSO) is larger than $\langle\alpha\rangle$ for the system characterized by an intact bilayer ( $7.5 \mathrm{~mol} \%$ of DMSO). The value of $\langle\alpha\rangle$ stays unchanged for DMSO concentrations ranging from 10 to $15 \mathrm{~mol} \%$ (Figure 4b), most likely because progressive redistribution of headgroups lining water pores is compensated by the overall more outward orientation of lipid headgroups due to membrane expansion.

It is noteworthy that for concentrations 12.5, 15.0, and 20.0 mol \% of DMSO, we witnessed formation of multiple pores. On average, we observed two transient water pores with an average pore radius of $\sim 1 \mathrm{~nm}$ in a membrane patch comprising an area of around $70 \mathrm{~nm}^{2}$. This leads us to an average pore density of $\sim 3 \times 10^{12}$ pore per $\mathrm{cm}^{2}$. In practical terms, for the example of a spherical liposome with a diameter of $1 \mu \mathrm{m}$, this means approximately $10^{5}$ pores, so that pores occupy around $10 \%$ of the overall liposome surface.

As seen from the mass density profiles (Figure 3), the structure of the system with $20 \mathrm{~mol} \%$ of DMSO deviates 

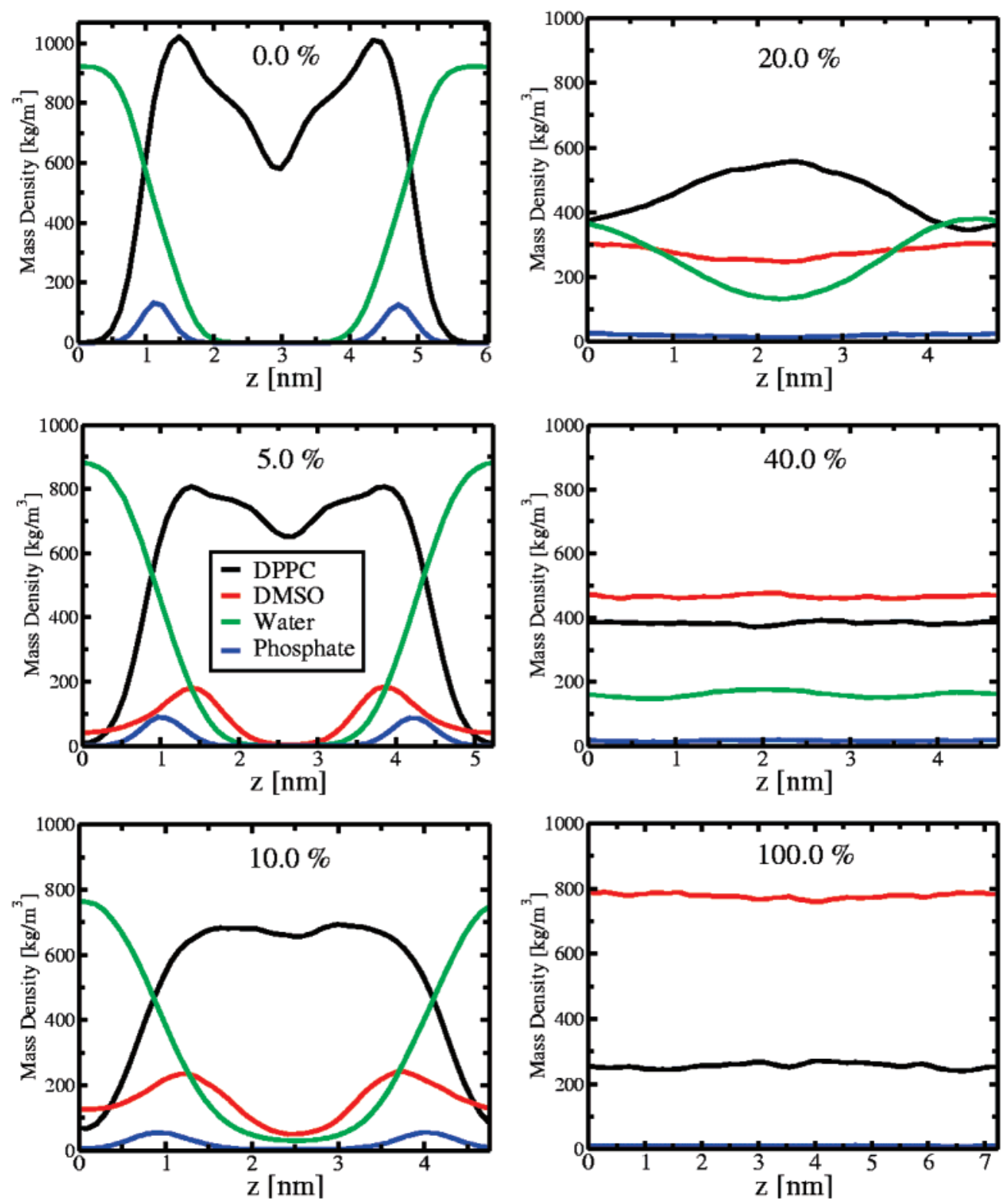

Figure 3. Componentwise mass density profiles for bilayer systems with $0,5,10,20,40$, and 100 mol $\%$ of DMSO. Shown are density profiles for DPPC lipids (black lines), DMSO molecules (red lines), water (green lines), and phosphorus atoms of lipid headgroups (blue lines).

significantly from that of a conventional lipid bilayer. The lipid/ water interface is smeared, large multiple pores take up considerable amount of water, and DMSO densities inside and outside the bilayer becomes indistinguishable. In fact, this system can be considered to be on the "borderline" of what could be defined as a bilayer. It turns out that a further increase in DMSO concentration fully destroys the bilayer structure and eventually leads to an isotropic structure comprising lipids, DMSO, and water (Figures 2 and 3). For this system, as would be expected, the acyl chains are almost fully disordered (the deuterium order parameter is close to zero), any preferential orientation of lipid headgroups is absent $(\langle\cos \alpha\rangle=0)$, and the interlipid coordination between the headgroups is at its minimal level (see Figure 4a).

Remarkably, we found that the early stages of membrane rupture are accompanied by desorption of individual lipids out of the membrane. Figure 6 illustrates this process for the DPPC bilayer system with $30 \mathrm{~mol} \%$ of DMSO. Lipid extraction events can be witnessed as early as within 4-5 ns after the onset of DMSO action. The lipid desorption observed here at an atomicscale resolution confirms some earlier suggestions based on experiments that this process may have a role in enhancing membrane permeability. ${ }^{23}$ Of notable interest is also the increase in curvature induced by DMSO of the lipid membrane as seen in Figure 6D.

A thorough consideration has to be given to possible artifacts related to the use of the periodic boundary conditions in our study: The progressive expansion and thinning of a membrane with DMSO concentration leads to a decrease in the repeat distance of a lamellar stack of periodic bilayer images, which is likely to enhance the bilayer-bilayer interaction effects. It is therefore very important to ensure that such "artificial" interaction does not affect the main findings of the present study. For these purposes we performed additional simulations of the bilayer systems at the three DMSO concentrations that were typical of each mode of DMSO's actions reported above, namely, 2.5, 10.0, and $30.0 \mathrm{~mol} \mathrm{\%}$; the number of solvent molecules (DMSO and water) in these simulations was increased by a factor of 4 . This ensures that one has around 6-8 nm between periodic images of a lipid bilayer, thereby minimizing the interaction between the bilayer images. It turns out that all our findings regarding modes of actions of DMSO also hold 
a

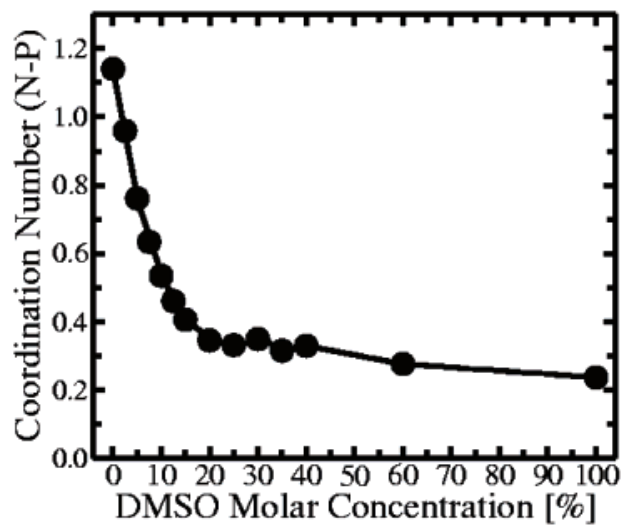

b

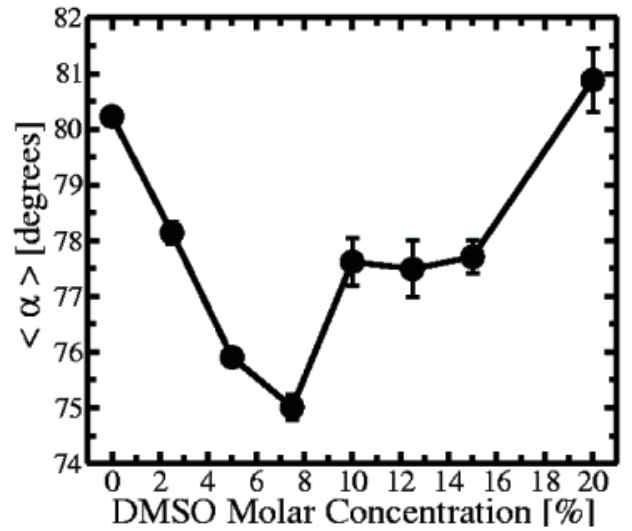

C

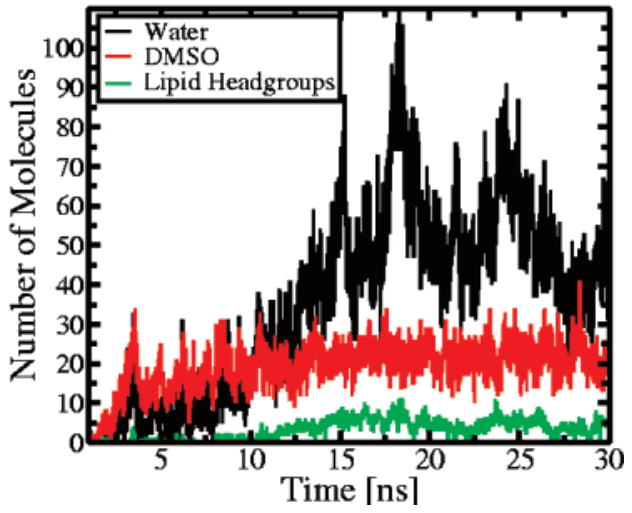

Figure 4. (a) Average coordination number of nitrogen atoms with phosphorus atoms of lipid headgroups as a function of DMSO molar concentration (only interlipid coordination was taken into account). (b) Average angle $\langle\alpha\rangle$ between the $\mathrm{P}-\mathrm{N}$ vector of DPPC lipid headgroups and the outward bilayer normal as a function of DMSO molar concentration. (c) The time evolution of numbers of water molecules (black), DMSO molecules (red), and lipid headgroups (green) within a $0.5 \mathrm{~nm}$ slab in the middle of the membrane during the pore formation process for the system with $12.5 \mathrm{~mol} \%$ of DMSO.

for these systems containing considerably increased amounts of solvent. The only significant difference is that we observe a certain enhancement in DMSO activity: DMSO-induced membrane thinning is now more pronounced, and both pore formation and membrane disintegration occur more rapidly compared to the smaller systems considered above. This is mostly due to the fact that the absolute amount of DMSO molecules approaching the membrane interface from the aqueous phase increases as the solvent/lipid ratio goes up. This, in particular, suggests that larger solvent/lipid ratios will shift the reported concentration ranges typical of different modes of DMSO activity to lower DMSO concentrations.

\section{Discussion}

The observed DMSO-induced thinning and expansion of a phospholipid bilayer and the increased fluidity of its hydrophobic core are able to explain why DMSO promotes the permeation of solutes, in particular hydrophobic entities, across the membrane: the path length is reduced and diffusion itself is facilitated because of the enhanced fluidity of the membrane interior. These findings are in good agreement with available X-ray diffraction measurements of DPPC bilayers in DMSO/water solvent. ${ }^{24}$ In that experimental study the authors found that DMSO at relatively low concentrations (up to $13.3 \mathrm{~mol} \%$ ) easily penetrates between the polar headgroups of phosphatidylcholine lipids and gives rise to an increase in the area occupied by lipid and to a drop in the bilayer thickness.

Probably the most significant finding of the present study is the induction of transient water pores by DMSO, confirming earlier suggestions from coarse-grained simulations. ${ }^{8}$ The formation of transient water pores in the current atomic-scale simulations spanning long timescales makes DMSO-induced pore formation a credible phenomenon. Furthermore, the implication of transient pore formation, i.e., enhanced permeability of hydrophilic solutes observed experimentally, ${ }^{25}$ links remarkably well with many of DMSO's pharmacological actions including analgesia, protection against ischemic injury, and cryopreservation, that involve modulation or disruption of ion or water transport across a cell membrane. It has to be emphasized that the fact that DMSO molecules do not interact favorably with lipid headgroups as discussed above is crucial for the observed pore formation as lipid molecules are not bound to each other through DMSO bridges and hence are able to reorient easily toward the membrane interior, stabilizing a pore. On this basis, one can expect a similar effect for other small aprotic solvents such as dimethylacetamide and dimethylformamide. ${ }^{23}$ On the other hand, polar protic solvents such as ethanol most likely do not induce formation of pores in membranes because of their strong hydrogen bonding to polar lipid headgroups.

The disruption of lipid bilayers observed in our study at high concentrations of DMSO suggests that DMSO is not able to hydrate a lipid bilayer in the way that water does. In particular, in the limiting case of pure DMSO solvent the structure of a bilayer was found to be destroyed (Figure 3), implying that a phospholipid bilayer cannot be stabilized by DMSO. This seems to be at odds with the electron density profiles observed in X-ray diffraction experiments that reveal evidence of a significantly disordered but interdigitated lamellar phase in pure DMSO solvent. ${ }^{26}$ However, these experimental studies refer to a gel phase structure at $T=303 \mathrm{~K}$, which is well below the $350 \mathrm{~K}$ temperature of the present study, and thus cannot directly be compared with the current results. Clearly, it would be both interesting and instructive to address this problem in the future by performing atomic-scale MD simulations studies of the effects of DMSO on phospholipid bilayers in the gel phase.

The various modes of action of DMSO provide a rationale for the experimentally observed action of DMSO as a fusogen of cells $s^{1,3}$ and of liposomes. As far as fusion of pure lipid membranes is concerned, the crucial step in the process is the merger of the proximal leaflets of the two bilayers. ${ }^{27}$ This step can be greatly facilitated by local perturbations of the structure of the proximal lipid leaflets and our findings indicate that DMSO fits well for this role: It induces pores, heavily perturbs the bilayer structure, and extracts lipids out of bilayer leaflets; all this can potentially lead to mixing up of lipids of proximal bilayers. 

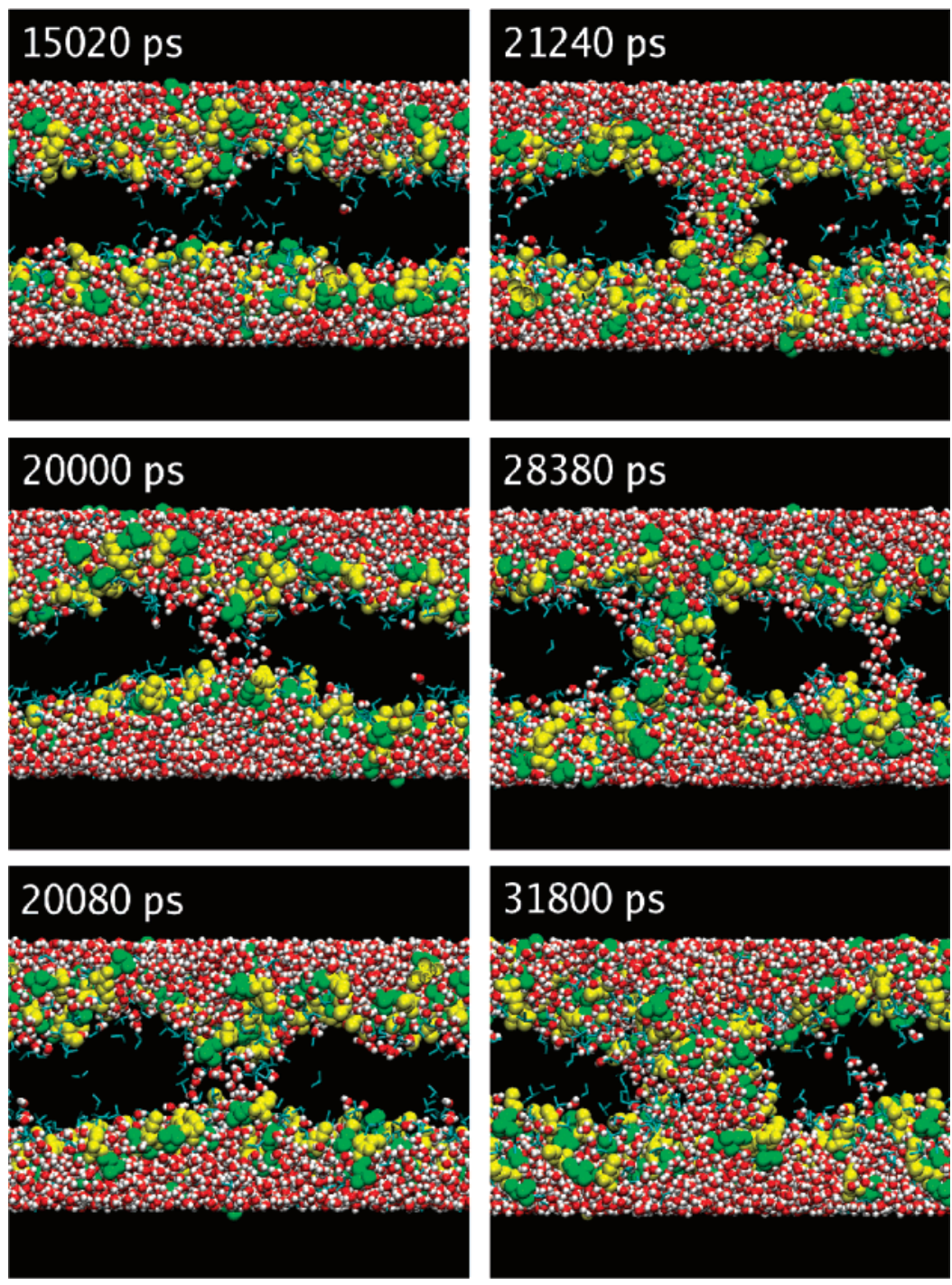

Figure 5. Pore formation in the bilayer system with $10 \mathrm{~mol} \%$ of DMSO. Water is shown in red, DMSO in cyan, choline and phosphate groups of lipid headgroups in green and yellow, respectively. Lipid acyl chains are not shown.

Now we turn to comparison of our findings with computational results of previous studies. In 1999 Smondyrev and Berkowitz reported an atomistic MD study of a DPPC bilayer in pure DMSO solvent at $T=323 \mathrm{~K}$ in which they found that only a very small number of DMSO molecules penetrated into the membrane so that the bilayer structure was stable and almost unaffected by DMSO. ${ }^{6}$ This result (superficially) contradicts both the experimental data and simulation studies reported later. ${ }^{7,8}$ This discrepancy is probably attributed to the very short (relative to current standards) simulation time employed in that study ( $2 \mathrm{~ns})$ and also to the lower temperature of the study, namely, $T=323 \mathrm{~K}$, which is much lower than the phase transition temperature of $350 \mathrm{~K}$ reported for the DPPC/DMSO system. ${ }^{15}$

The study of Sum and de Pablo ${ }^{7}$ is more comprehensive, investigating the effect of DMSO at various concentrations. At low DMSO concentrations $(<10 \mathrm{~mol} \%)$ their results are in very good agreement with our findings. However, at the $10 \mathrm{~mol} \%$ DMSO they did not find any evidence that water molecules can penetrate into the bilayer for the system, whereas we report formation of a water pore at this DMSO concentration. We believe the reason for this discrepancy is twofold. First, again the simulation time is considerably shorter, $13 \mathrm{~ns}$ versus our $50 \mathrm{~ns}$ (we recall that formation of a stable water pore at $10 \mathrm{~mol}$ $\%$ is observed only after $20 \mathrm{~ns}$ ). Second, the difference in force- fields employed for the DPPC lipids between the studies could have a significant effect. For a DPPC bilayer without DMSO at $T=350 \mathrm{~K}$, Sum and de Pablo reported $0.668 \mathrm{~nm}^{2}$ for the area per lipid $\langle A\rangle$, whereas we found a larger value of $0.69 \mathrm{~nm}^{2}$ (see Table 1). In particular, this indicates that the force-field used in our study provides a better match with the experimental value of $0.719 \mathrm{~nm}^{2}$ at $T=353 \mathrm{~K}^{18}$ More importantly, this suggests that the force-field used by Sum and de Pablo favors a more condensed state of the DPPC bilayer compared to our model. Indeed, for the system with $10 \mathrm{~mol} \%$ of DMSO they found $^{7}$ an area per lipid of $\langle A\rangle=0.87 \mathrm{~nm}^{2}$; this area approximately corresponds in our case to a bilayer system with a considerably lower concentration of DMSO (between 5 and $7.5 \mathrm{~mol} \%$, see Table 1). Therefore, in a certain sense, our findings are in line with the results reported by Sum and de Pablo: there is no pore formation observed for a DPPC/DMSO/ water system with an area per lipid of $\sim 0.9 \mathrm{~nm}^{2}$. This means that the potential model employed in ref 7 will most likely also reveal pore formation for more expanded bilayers, i.e., at higher DMSO concentrations.

For the pure DMSO solvent system, Sum and de Pablo's results $^{7}$ are in contrast to current results in that they found the DPPC bilayer to be stable, whereas the bilayer disintegrates in the current study. However, a closer inspection of their results reveals that there is no actual discrepancy with our results. They 

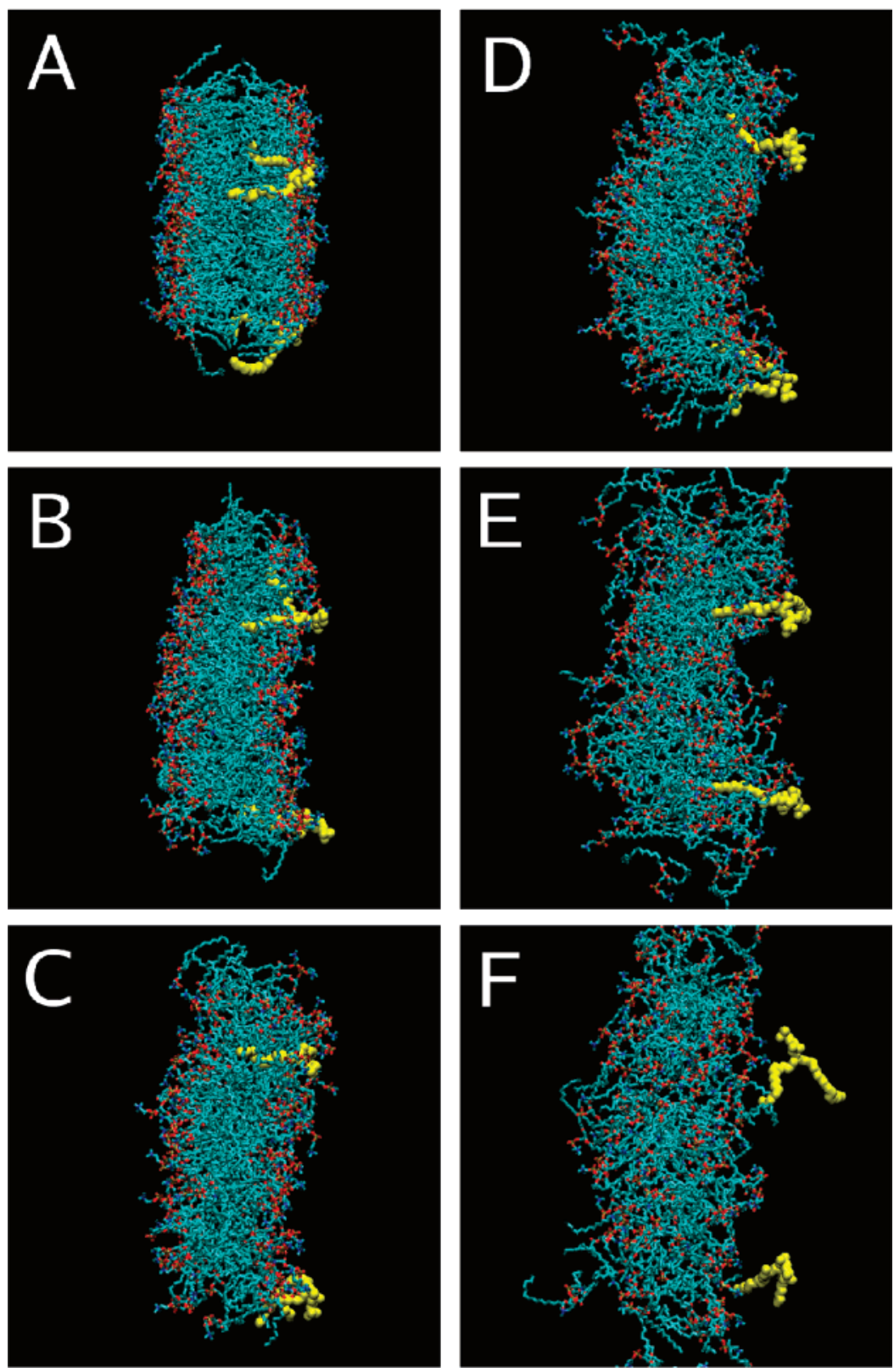

Figure 6. Desorption of lipid molecules from a membrane under the influence of $30 \mathrm{~mol} \%$ of DMSO: (A) 0 ps, (B) $2280 \mathrm{ps,} \mathrm{(C)} 3030 \mathrm{ps,} \mathrm{(D)}$ 3400 ps, (E) 4500 ps, and (F) 9490 ps. Extracted lipids are highlighted in yellow; water and DMSO are not shown.

report a markedly lower area per lipid for the pure DMSO solvent system compared with their 10 mol \% DMSO system $\left(77.3 \mathrm{vs} 87.0 \mathrm{~nm}^{2}\right)$. They also observe that the bilayer interface is not well defined and that the acyl chains are much more disordered with at least some of them exposed to the bulk DMSO solvent. This description of the state of the DPPC bilayer in pure DMSO is akin to the early stages of bilayer disintegration observed by us. We consider that Sum and de Pablo's system would also disintegrate given more time and possibly a greater number of DMSO molecules. Their force-field, which favors a more condensed system, is also likely to add some additional inertia to system disintegration.

In conclusion, we have performed a systematic study of the effects of the aprotic solvent, dimethyl sulfoxide, on phosphatidylcholine lipid membranes at $T=350 \mathrm{~K}$ using atomic-scale MD simulations. DMSO exhibits three distinct modes of action, each in a particular concentration regime. At low concentration (below $10 \mathrm{~mol} \%$ ), DMSO induces significant lateral expansion of the membrane with a concurrent drop in membrane thickness. At DMSO concentrations in the range from 10 to $20 \mathrm{~mol} \%$, we witness formation of transient water pores and water defects in addition to progressive thinning of the membrane. Further increase in DMSO concentration leads to the desorption of individual lipid molecules out of the membrane surface followed by disintegration of the bilayer structure of the lipid membrane. The reported membrane thinning and, in particular, pore formation provide a credible molecular-level explanation of how DMSO promotes permeation of molecules, particularly those of hydrophilic nature, through lipid membranes as observed experimentally. The DMSO-induced water pores and associated enhanced permeability is remarkably consistent with many of DMSO's pharmacological effects (e.g., analgesia, protection against ischemic injury, and cryopreservation), which on the basis of current findings can all be explained by modulation or disruption of solute transport across membrane via DMSOinduced pore formation. Furthermore, the local loss in integrity of the bilayer structure of lipid membranes induced by high concentrations of DMSO could be a key for understanding the molecular mechanism that enables DMSO to be a very effective cell fusogen. Finally, the study emphasizes the importance of 
identifying an optimal concentration of DMSO in practical applications in cell biology, since different applications (both current and new) would depend critically on DMSO acting in a particular regime.

\section{References and Notes}

(1) Ahkong, Q. F.; Fisher, D.; Tampion, W.; Lucy, J. A. Nature 1975, 253, 194-195.

(2) Lyman, G. H.; Priestler, H. D.; Papahadjopoulos, D. Nature 1976, $262,360-363$.

(3) Anchordoguy, T. J.; Carpenter, J. F.; Crowe, J. H.; Crowe, L. M. Biochim. Biophys. Acta 1992, 1104, 117-122.

(4) Rall, W. F.; Fahy, G. M. Nature 1985, 313, 573-575.

(5) Yu, Z.-W.; Quinn, P. J. Biosci. Rep. 1994, 14, 259-281. 2478 .

(6) Smondyrev, A. M.; Berkowitz, M. L. Biophys. J. 1999, 76, 2472-

(7) Sum, A. K.; de Pablo, J. Biophys. J. 2003, 85, 3636-3645.

(8) Notman, R.; Noro, M.; O'Malley, B.; Anwar, J. J. Am. Chem. Soc. 2006, 128, 13982-13983. 2013.

(9) Berger, O.; Edholm, O.; Jahnig, F. Biophys. J. 1997, 72, 2002-

(10) Berendsen, H. J. C.; Postma, J. P. M.; van Gunsteren, W. F.; Hermans, J. In Intermolecular Forces; Pullman, B., Ed.; Reidel: Dordrecht, The Netherlands, 1981; p 331.

(11) Bordat, P.; Sacristan, J.; Reith, D.; Girard, S.; Glattli, A.; MullerPlathe, F. Chem. Phys. Lett. 2003, 374, 201-205.

(12) Darden, T.; York, D.; Pedersen, L. J. Chem. Phys. 1993, 98, 1008910092
(13) Essman, U.; Perera, L.; Berkowitz, M. L.; Darden, T.; Lee, H.; Pedersen, L. G. J. Chem. Phys. 1995, 103, 8577-8592.

(14) Berendsen, H. J. C.; Postma, J. P. M.; van Gunsteren, W. F.; DiNola, A.; Haak, J. R. J. Chem. Phys. 1984, 81, 3684-3690.

(15) Shashkov, S. N.; Kiselev, M. A.; Tioutiounnikov, S. N.; Kiselev, A. M.; Lesieur, P. Physica B 1999, 271, 184-191.

(16) Lindahl, E.; Hess, B.; van der Spoel, D. J. Mol. Model. 2001, 7, $306-317$.

(17) Nagle, J. F.; Tristram-Nagle, S. Biochim. Biophys. Acta 2000, 1469, 159-195.

(18) Petrache, H. I.; Dodd, S. W.; Brown, M. F. Biophys. J. 2000, 79, $3172-3192$.

(19) Pasenkiewicz-Gierula, M.; Takaoka, Y.; Miyagawa, H.; Kitamura, K.; Kusumi, A. Biophys. J. 1999, 76, 1228-1240.

(20) Gurtovenko, A. A.; Patra, M.; Karttunen, M.; Vattulainen, I. Biophys. J. 2004, 86, 3461-3472.

(21) Tieleman, D. P.; Leotiadou, H.; Mark, A. E.; Marrink, S.-J. J. Am Chem. Soc. 2003, 125, 6382-6383.

(22) Gurtovenko, A. A.; Vattulainen, I. J. Am. Chem. Soc. 2005, 127 , $17570-17571$.

(23) Barry, B. W. Nat. Biotechnol. 2004, 22, 165-167.

(24) Yu, Z.-W.; Quinn, P. J. Biophys. Chem. 1998, 70, 35-39.

(25) Williams, A. C.; Barry, B. W. Adv. Drug Delivery Rev. 2004, 56, $603-618$

(26) Gordeliy, V. I.; Kiselev, M. A.; Lesieur, P.; Pole, A. V.; Teixeira, J. Biophys. J. 1998, 75, 2341-2351.

(27) Jahn, R.; Grubmuller, H. Curr. Opin. Cell Biol. 2002, 14, 488- 